\title{
Effects of corrective insole on leg muscle activation and lower extremity alignment in rice farmers with pronated foot: a preliminary report
}

\author{
Agung Kristanto ${ }^{\mathrm{a}, \mathrm{c}}$, Manida S. Neubert ${ }^{\mathrm{b}, \mathrm{c}, *}$, Michael T. Gross ${ }^{\mathrm{d}}$, Rungthip Puntumetakul ${ }^{\mathrm{c}, \mathrm{e}}$, \\ David B. Kaber ${ }^{\mathrm{f}}$, Weerapat Sessomboon ${ }^{\mathrm{a}}$
}

a Department of Industrial Engineering, Faculty of Engineering, Khon Kaen University, Khon Kaen, Thailand

${ }^{\mathrm{b}}$ Program of Production Technology, Faculty of Technology, Khon Kaen University, Khon Kaen, Thailand

${ }^{\mathrm{c}}$ Research Center in Back, Neck, Other Joint Pain and Human Performance (BNOJPH), Khon Kaen, Thailand

d Division of Physical Therapy, Department of Allied Health Sciences, University of North Carolina at Chapel Hill, NC, USA

e School of Physical Therapy, Faculty of Associated Medical Sciences, Khon Kaen University, Khon Kaen, Thailand

${ }^{\mathrm{f}}$ Department of Industrial and Systems Engineering, University of Florida, Gainesville, FL, USA

\section{A R T I C L E I N F O}

\section{Keywords:}

Medial wedge insole

Muscle activity

Foot pronation

Lower extremity alignment

Working surface condition

\begin{abstract}
A B S T R A C T
Background: Execution of strenuous activities in conjunction with slippery and viscous muddy working terrain in rice cultivation leads to a high prevalence of farmer musculoskeletal disorders and malalignments. Recommended intervention strategies originally designed for congenitally disabled individuals may also be applicable to farmers, including simple corrective wedges to reduce foot eversion. The objective of the present study was to conduct a preliminary investigation of the effects of corrective wedges on lower extremity muscle activity and alignment when subjects stood on flat rigid ground or muddy terrain, simulating typical work conditions encountered by the unique but populous Thai rice farming workforce.

Methods: Nine healthy farmers with pronated feet were recruited to participate and wedges were custom fabricated for each farmer based on physical therapy assessment and use of rapid prototyping techniques. Participants were asked to stand barefoot or with wedges on the two surface types.

Results: Results revealed foot pronation and knee valgus to improve (ranging, on average between 5.5 and 16.1 degrees) when participants were equipped with corrective wedges. The muscle activity of the peroneus longus and the tibialis anterior increased for muddy terrain, as compared with the rigid surface. In general, the wedges induced less tibialis anterior activity and greater peroneus longus activity, compared to when participants were standing barefoot. An elevation in evertor muscle activity may reflect stretching of the shortened muscle as a result of the reduced degree of foot pronation.

Conclusions: Findings demonstrate potential benefits of corrective insole usage for farmers with pronated feet, including improved lower extremity alignment and invertor muscle activity reduction for both rigid and muddy terrains.
\end{abstract}

\section{Introduction}

The extreme environment and strenuous work requirements of certain rice cultivation processes causes farmers to develop a high prevalence of musculoskeletal disorders (MSDs) and malalignments in various body parts [1,2]. In a recent study [3], foot pronation, which is defined by Horwood and Chockalingam [4] as a range of motion within the foot that makes the foot more prone to the support surface, was identified as the predominant disorder among rice farmers in Khon Kaen, Thailand, with a prevalence of $36.1 \%$. Excessive foot pronation may be greater than that required by the individual to adjust to morphology or forces imposed on the musculoskeletal system by gait or another action. Such abnormal body alignments may occur in rice farmers due to working conditions in rice paddy fields. A previous investigation of risk factors showed that years of farming experience strongly correlated with the specific type of foot malalignment [3]. Rice farming in Thailand is primarily comprised of manual labor (National Statistical Office), including frequent lifting of heavy loads with awkward postures and prolonged standing on both muddy and flat rigid terrain [5]. Progressive structural deformity of weight-bearing foot

\footnotetext{
* Corresponding author at: Program of Production Technology, Faculty of Technology, Khon Kaen University, Khon Kaen 40002, Thailand.

E-mail address: manida@kku.ac.th (M.S. Neubert).
} 


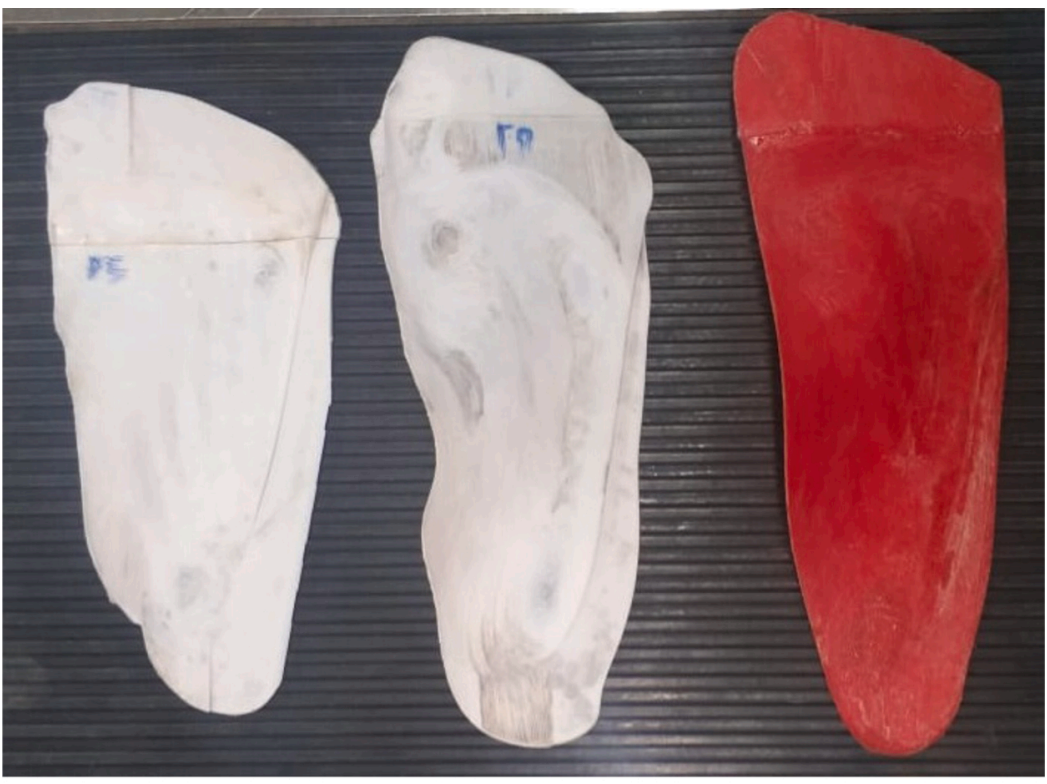

(A)

(B)

(C)

Fig. 1. Examples of medial wedge insole design of sizes: (A) $10^{\prime \prime}$ (B) $10.5^{\prime \prime}$; and (C) $11^{\prime \prime}$.

pronation in rice farmers might be caused by compensation for foot instability and control of body alignment. Such conditions frequently occur during prolonged activities in the stance phase on slippery and highly viscous mud, which causes adverse effects between ground reaction forces and abnormal rotational alignment of the lower extremity [6]. In addition, the preference of farmers to perform work with bare feet is suspected to aggravate MSDs and lower limb malalignments [7].

Abnormal foot structures can further raise the risk of lower limb injuries $[8,9]$ while abnormalities in foot pronation might result in passive hypermobility and instability of specific joints [10]. Consequently, individuals suffering from pronated feet may feel unstable during weight bearing, demonstrate poorer balance, and demonstrate a higher risk of injury due to slipping or falling [11]. In addition, there is evidence of a close relationship between variations in foot structure among healthy individuals and the risk of lower limb injury, possibly due to changes in lower limb muscle activity [12]. Foot pronation can drive abnormal rearfoot eversion of the hindfoot causing abnormal ankle joint and subtalar joint loading. This same research indicated individuals with pronated feet depend on additional muscular support while walking or running [12] and induced fatigue can increase the risk of injury [13]. As foot pronation and knee valgus are linked deformities, foot pronation can also drive abnormal internal rotation at the knee and the hip. In addition, foot pronation can drive knee valgus loading and the condition can cause arch collapse thereby creating abnormal tensile stress of the plantar fascia and the tibialis posterior muscle-tendon unit. However, despite knowledge and general awareness of the above hazards, there have been few successful intervention designs for rice farmers [14,15].

Our previous study reviewed and recommended several ergonomic interventions, originally designed for disabled populations, but having applicability to rice farmers [16]. The study revealed most existing and proposed interventions for farmers are based on educational programs and tool redesigns. There has also been no attempt to adapt readily available interventions for congenitally disabled populations for the healthy farmer workforce. Previous research [16-21] has indicated that orthotic devices, including molded foot orthoses, removable external orthotic devices, and inserts or custom designed insoles, could have great value for treatment of foot pronation. Although some earlier devices provide additional correction of foot pronation in congenitally disabled populations, they also present certain drawbacks that render them significantly less applicable for rice farmers. Molded orthotics are expensive and require many manufacturing steps in production of structural elements. Moreover, molded orthotics are difficult to fit into existing footwear due to bulkiness of the material [22]. Removable external orthotic devices (e.g., ankle foot orthoses) may restrict movement due to rigid designs $[18,19]$. In rice farming, there is frequent forward and backward walking in conjunction with whole body bending and twisting. Hence, devices imposing restrictions on movement would be unsuitable for the paddy environment. More recent designs of orthotics with adaptive control systems have become available; however, they are expensive and require an external power source $[18,19]$. Given the socioeconomic conditions and infrastructure limitations of the Thai agricultural society, such costly, power-dependent devices are not feasible. A simple wedge has been proposed as an alternative treatment device and is often selected by medical practitioners to provide patients with direct therapy. Wedges can be used in footwear where space is restricted and require significantly less manufacturing time (up to 3 times less) and cost up to 2.5 times less, as compared with molded orthotics [21]. Recent advances in custom orthotic production make use of 3D-scanning technology to acquire models of patient feet, as well as rapid prototyping to accelerate the orthotic production process $[23,24]$. This procedure allows custom devices to be quickly designed and manufactured and, therefore, represents a highly practical means of development of interventions the rice farming workforce.

Interventions for the unique and populous rice farmer workforce are urgently needed due to work and environmental hazards. To date, there has been no prior study applying corrective wedges as an ergonomic intervention aimed at preventing or reducing musculoskeletal injury in a group of rice farmers working in a paddy field. The objective of the present study was to conduct a preliminary investigation of the effects of simple corrective wedges on farmer leg muscle activity and foot and knee alignments, induced by standing on different work terrain surfaces, including normal flat rigid ground and a muddy surface. These two types of terrain conditions are typically encountered by farmers in cultivation tasks. It was hypothesized that muddy terrain would induce greater deviation of knee alignment and muscle activity related to foot inversion and eversion (i.e., tibialis anterior (TA) and peroneus longus (PL), respectively), as farmers require compensation for foot instability and 


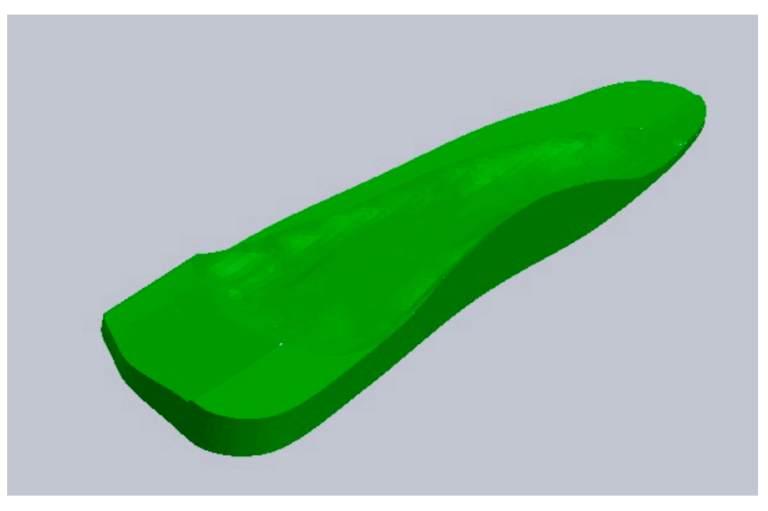

Fig. 2. Example of a $3 \mathrm{D}$ model for a complete design of the medial wedge insole.

control of body alignment while standing in the mud. A proposed medial wedge insole was expected to correct deviations of foot and knee alignments and reduce muscle activity for both terrain conditions. The study was expected to provide a preliminary demonstration of applicability of orthotic devices, which are primarily used by disabled populations, for extended use by the agricultural workforce.

\section{Materials and methods}

\subsection{Participants}

Experienced rice farmers (males and females aged between 20 and 59 years with 1 year or more field time) were recruited from Khon Kaen Province, Thailand. Participants were also required to have no prior medical history affecting lower extremity alignment, such as surgery and/or a fracture. Subsequently, rear foot and medial longitudinal arch angles were measured for all participants, based on Jonson and Gross's method [25]. The method was selected because it is quick and easy and simultaneously offers numerical criteria for foot state classification. Notably, the method also has acceptable intra-rater (ICC $=0.88-0.9$ ) and inter-rater reliability (ICC $=0.81-0.86$ ). Participants were included in the study based on diagnosis of foot pronation (i.e., a rear foot angle greater than 9 degrees and a medial longitudinal arch angle less than 134 degrees). We recruited farmers with foot pronation to investigate changes in lower extremity alignment and muscle activity vs. correcting their disability. The study sample size was based on previous significant studies with sample sizes ranging from 9 to 70 subjects [20,21]. The study was approved by a local Ethics Committee for Human Research.

\subsection{Corrective insole design and fabrication}

Corrective insoles were custom designed and manufactured for each participant. The size of the insoles was determined based on participant shoe sizes, which was either $10^{\prime \prime}, 10.5^{\prime \prime}$, or $11^{\prime \prime}$ (Fig. 1). The design of the medial wedges was based on a 3D optical scan of the foot and a clinical examination conducted by physical therapists. A Sense 3D scanner (3D Systems, Inc., Rock Hill, USA) was used for acquisition of a 3D image of each participant's foot while the subject was seated with their legs held out horizontally, supported on a stable chair of the same height. The 3D image of the foot was used to create a 3D computer-based model of the insole and a custom filling of the arch using the SolidWorks software package (Dassault Systèmes SolidWorks Corporation, Waltham, USA). Based on clinical examination and measurement of foot malalignment (see section on measurement of lower extremity alignment), the specific height and angle of the medial forefoot wedge, medial rear foot wedge and heel lift (required to correct the individual foot alignment) were identified and incorporated in the model. Specifically, an 1/8-inch $(0.318 \mathrm{~cm})$ heel lift was prescribed if participants exhibited less than neutral dorsiflexion at the ankle with the knee extended. Inadequate dorsiflexion at the talocrural joint can lead to dorsiflexion and other components of pronation at the subtalar joint. A medial forefoot wedge, with the angular magnitude of measured forefoot varus, was included if the participant exhibited forefoot varus more than or equal to 5 degrees.
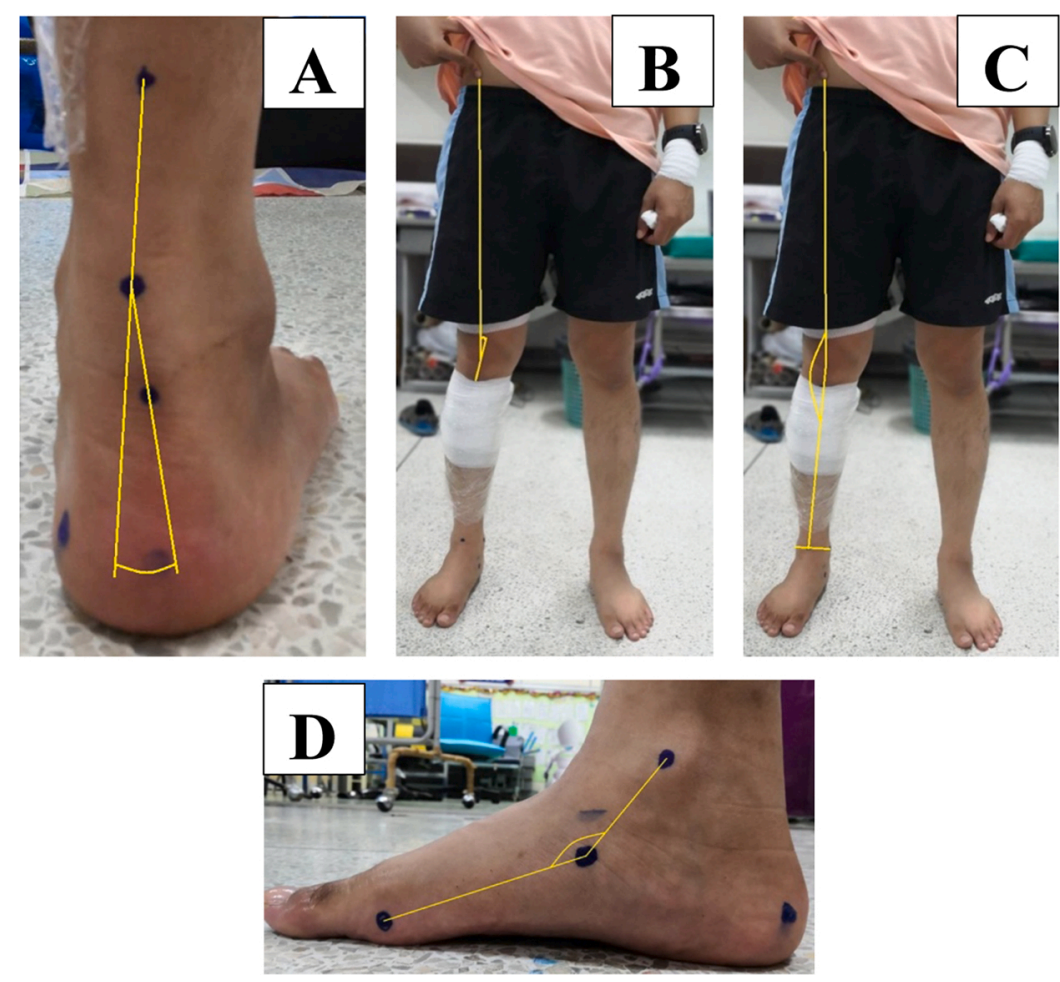

Fig. 3. Lower extremity alignment measurement methods for: (A) rear foot angle; (B) quadriceps angle; (C) tibiofemoral angle and; (D) medial longitudinal arch angle. 


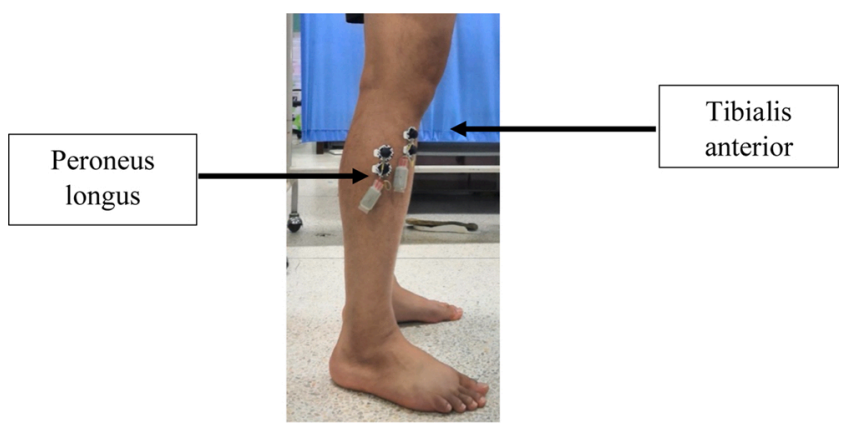

Fig. 4. Illustration of EMG electrode placements of peroneus longus and tibialis anterior.

This wedge shifted ground reaction force medially at initial contact of the forefoot, thereby driving less compensatory eversion at the subtalar joint. If the rearfoot angle of the participant was more than 9 degrees, a medial rearfoot wedge was included. The wedge corrected the rearfoot angle to be less than 9 degrees but did not exceed an 1/8-inch $(0.318 \mathrm{~cm})$ height. The medial rearfoot wedge was provided to promote a smaller rearfoot to leg angle, thereby placing the subject's subtalar joint in a less everted or pronated position. Fig. 2 shows an example of a 3D computer-based model for a complete design of the corrective insole. Once the design was completed, the wedge insole was manufactured by printing polylactic acid (PLA) filaments on a FlashForge 3D Printer Creator Pro (Zhejiang Flashforge 3D technology Co., LTD, Jinhua, China).

\subsection{Measurement of lower extremity alignment}

The alignments of rear foot angle, medial longitudinal arch angle, quadriceps $(Q)$ angle, and tibiofemoral angle were measured in this study. The measurements were conducted in a standardized standing position and were based on the methods described in ([3], Fig. 3). All measurements were repeated 3 times by a single physical therapist.
Participants' feet were classified as showing normal alignment when the rear foot angle ranged between 3 and 9 degrees and the medial longitudinal arch angle ranged between 134 and 150 degrees [25]. A genu valgus (knock-knee) was identified when the Q-angle was greater than 18 degrees or the tibiofemoral angle was less than 173 degrees. For normal knee alignment, the Q-angle of males and females is approximately 10-13 degrees and 15-18 degrees, respectively, and the tibiofemoral angle is approximately $173-180$ degrees $[26,27]$.

\subsection{Measurement of lower extremity muscle activity}

The foot invertor and evertor muscles, TA and PL, respectively, were connected to a wireless electromyograph (EMG; Wave Plus, Cometa, Milan, Italy), after which the EMG Easy Report software (MerloBioEngineering, Parma, Italy) was used to measure and analyze muscle activity. To reduce impedance, the skin underlying the electrodes was shaved, scraped with sandpaper, and cleaned with alcohol. Two pairs of surface EMG electrodes were placed $2 \mathrm{~cm}$ apart over anatomical locations of TA and PL muscles, based on the guidelines from previous research [28]. Specifically, the electrodes were placed $3 \mathrm{~cm}$ below the fibular head to measure PL muscle activity, and $1 \mathrm{~cm}$ lateral to the tibia edge and $8 \mathrm{~cm}$ below the tibial tuberosity to measure TA muscle activity (Fig. 4). Muscle activity of the TA and the PL was collected simultaneously from the participants' right leg at a sampling rate of $2000 \mathrm{~Hz}$. EMG data were filtered using $20-500 \mathrm{~Hz}$ bandpass and $50 \mathrm{~Hz}$ notch filters. The root mean square (RMS) values were obtained over the 1-min standing duration, and normalized as an average percentage of the maximal voluntary isometric contraction (\%MVIC). Based on guidelines from previous studies, the MVIC value of the PL and TA were measured in a seated position with the knee and ankle positioned at $90^{\circ}$ angle and feet placed on a platform $[29,30]$. For PL, a constraint band was passed over the foot while participants were asked to perform plantar flexion and ankle eversion. For TA, a constraint band was passed over the knee while participants were asked to perform plantar flexion and dorsiflexion of the forefoot.
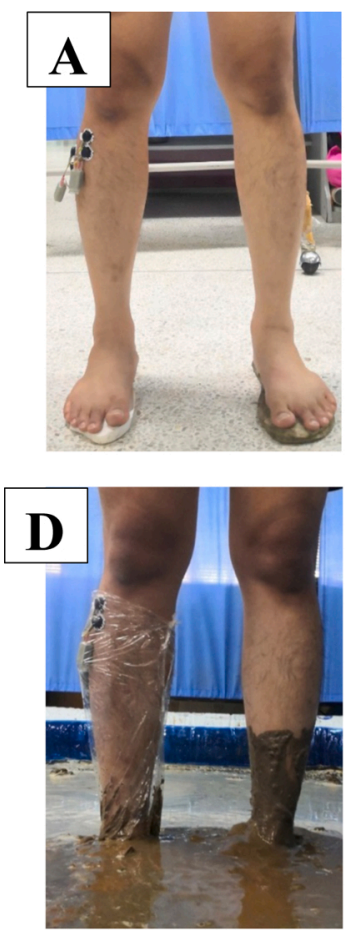
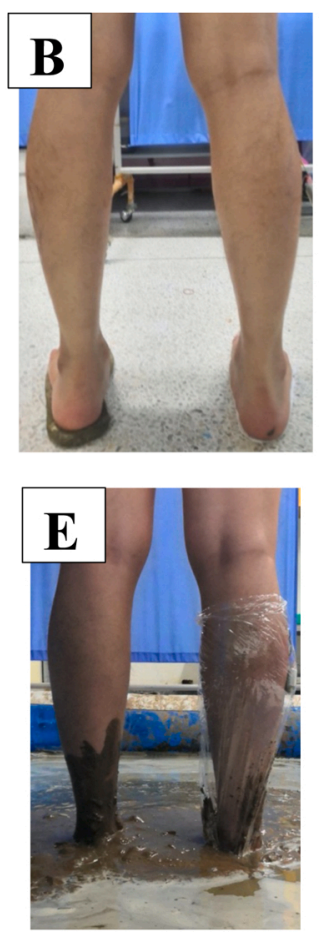
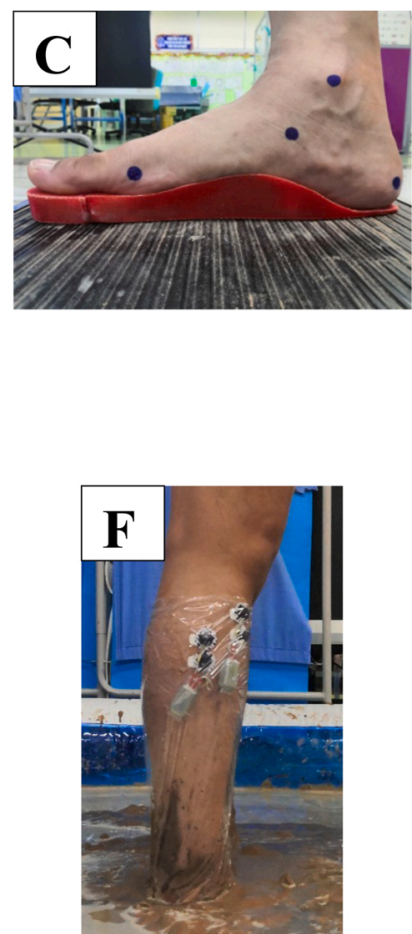

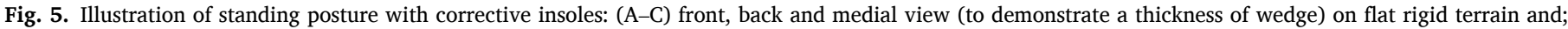
(D-F) front, back and lateral view (due to a difficulty to obtain the medial side of foot) on muddy terrain. 
Table 1

Participants characteristics.

\begin{tabular}{|c|c|c|}
\hline Characteristics & $n(\%)$ & Mean (SD) \\
\hline \multicolumn{3}{|l|}{ Gender } \\
\hline Male & $4(44.44)$ & \\
\hline Female & $5(55.56)$ & \\
\hline Age (years) & & $51.89(6.86)$ \\
\hline Height (cm) & & $161.78(6.10)$ \\
\hline Weight (kg) & & $59.80(11.70)$ \\
\hline Experience (years) & & $36.11(9.71)$ \\
\hline \multicolumn{3}{|l|}{ Size of footwear } \\
\hline Size 10 & $1(11.11)$ & \\
\hline Size 10.5 & $6(66.67)$ & \\
\hline Size 11 & $2(22.22)$ & \\
\hline Rearfoot angle (deg) & & $10.70(1.57)$ \\
\hline Normal & $0(0)$ & \\
\hline Abnormal & $9(100)$ & \\
\hline Medial longitudinal arch angle (deg) & & $128.65(2.38)$ \\
\hline Normal & $0(0)$ & \\
\hline Abnormal & $9(100)$ & \\
\hline Q-angle (deg) & & $21.49(1.88)$ \\
\hline Normal & $0(0)$ & \\
\hline Abnormal & $9(100)$ & \\
\hline Tibiofemoral angle (deg) & & $170.82(2.31)$ \\
\hline Normal & $0(0)$ & \\
\hline Abnormal & $9(100)$ & \\
\hline
\end{tabular}

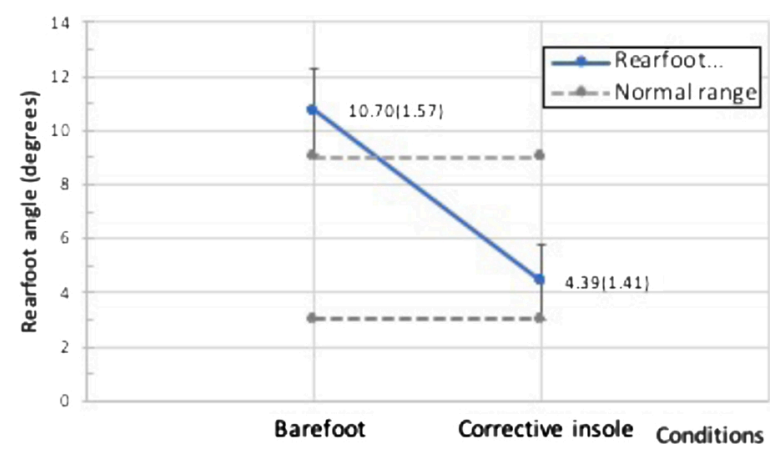

Fig. 6. Results of rearfoot angle alignment for rigid work terrain condition.

\subsection{Experimental procedure}

The study consisted of two footwear conditions, (1) barefoot (BF) and (2) with corrective insole (CI); and two work terrain surfaces, (1) flat rigid and (2) muddy surface. For the latter condition, a mud-filled container with a flat bottom surface was used. The order of participant exposure to the experimental conditions was randomized. They were initially instructed to assume a standing position with feet shoulder-width apart, maintaining a stable posture, and looking straight ahead for $1 \mathrm{~min}$. In this position, lower extremity alignments were measured and EMG data was recorded. Due to limited visibility, the foot alignments, including rear foot angle and medial longitudinal arch angle, could not be measured when participants were standing in the mud. Fig. 5 illustrates a participant equipped with corrective insoles standing on the two work terrain conditions. Based on a recommendation from prior research, all participants were provided a 5-min break between each experiment condition to relieve any potential muscle fatigue [31]. To prevent potential injury through falling or slipping, the corrective insoles were secured by plastic wraps to the participants' feet while standing on muddy terrain.

\section{Results}

\subsection{Demographic data of participants}

Relevant participant characteristics are displayed in Table 1. The age

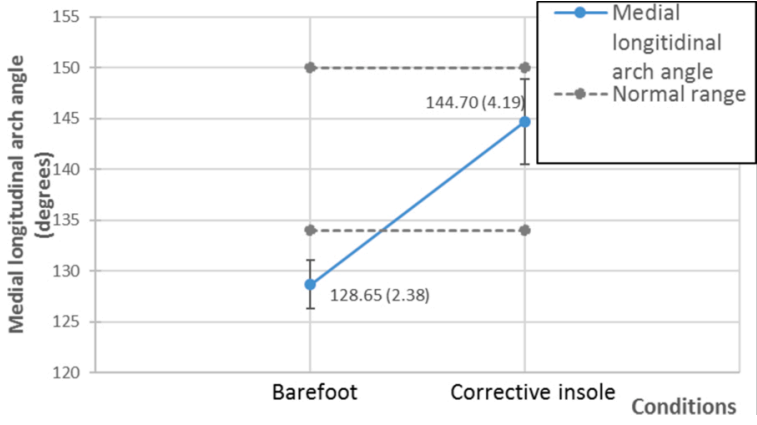

Fig. 7. Results of medial longitudinal arch angle alignment for rigid work terrain condition.

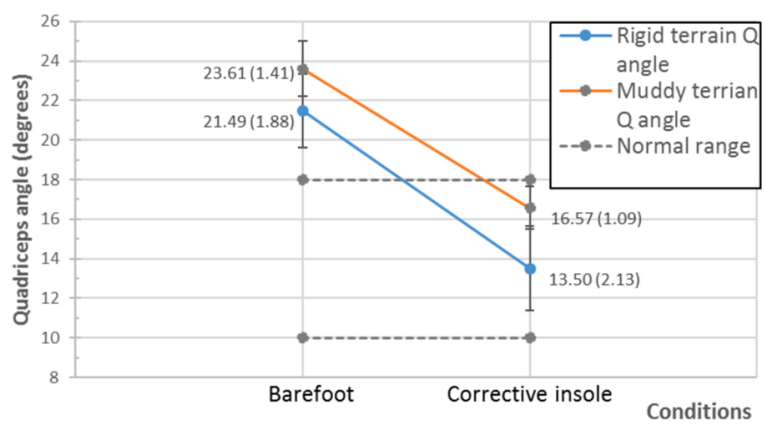

Fig. 8. Results of Q-angle alignment for various experimental terrain conditions.

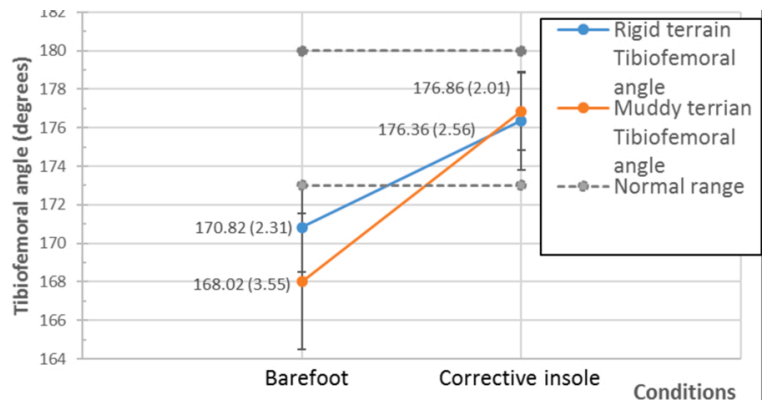

Fig. 9. Results of tibiofemoral angle alignment for various experimental terrain conditions.

of the participants ranged from 40 to 62 years and their experience in rice farming ranged from 15 to 49 years. Most participants wore $10.5^{\prime \prime}$ footwear. All participants exhibited excessive foot pronation identified by abnormal rear foot and medial longitudinal arch angles, as well as excessive knee valgus identified by abnormal $\mathrm{Q}$ or tibiofemoral angles.

Table 2

Mean (SD) of muscle activity involved during flat and muddy terrain standing position.

\begin{tabular}{llllll}
\hline \multirow{2}{*}{$\begin{array}{l}\text { Muscle activity (\% } \\
\text { MVIC) }\end{array}$} & \multicolumn{4}{c}{ Conditions (mean (SD)) } \\
\cline { 2 - 3 } & \multicolumn{2}{c}{ Barefoot (BF) } & & \multicolumn{2}{c}{ Corrective insole (CI) } \\
\cline { 2 - 3 } \cline { 5 - 6 } & $\begin{array}{l}\text { Rigid } \\
\text { terrain }\end{array}$ & $\begin{array}{l}\text { Muddy } \\
\text { terrain }\end{array}$ & & $\begin{array}{l}\text { Rigid } \\
\text { terrain }\end{array}$ & $\begin{array}{l}\text { Muddy } \\
\text { terrain }\end{array}$ \\
\hline Tibialis anterior (TA) & $0.80(0.22)$ & $1.32(0.20)$ & & $0.69(0.19)$ & $1.04(0.21)$ \\
Peroneus longus (PL) & $1.01(0.19)$ & $1.69(0.16)$ & & $2.43(0.23)$ & $2.59(0.41)$ \\
\hline
\end{tabular}




\section{Brief summary}

What is already known

- The prevalence of MSDs and malalignments among rice farmers in Thailand has been found to be very high.

- Interventions for the rice farmer workforce are urgently needed as a result of work and environmental hazards.

- Previous studies have suggested intervention strategies for individuals with disabilities to have utility for preventing occupation-related injuries in healthy individuals.

What this study adds

- Foot pronation and knee valgus improved when rice farmers were equipped with corrective wedges.

- Wedges induce lower tibialis anterior activity and greater peroneus longus activity, as compared to barefoot standing.

- Preliminary investigation demonstrates corrective insole usage to improve alignment and reduce invertor muscle force.

\subsection{Lower extremity alignment}

Figs. 6-9 present the angle measurements for lower extremity alignment of participants under the varied terrain and footwear conditions.

\subsection{Lower extremity muscle activity}

Table 2 presents a comparison of the average percentage of \%MVIC of PL and TA muscle activity between BF and CI conditions during standing on flat rigid and muddy terrains.

\section{Discussion}

The present study represents a preliminary report on the efficacy of simple corrective insoles on foot muscle activity as well as foot and knee alignments of farmers with pronated feet when standing on rigid flat and muddy surfaces.

\subsection{Corrective insole design and fabrication}

A corrective medial wedge was selected for investigation due to its practicality for use by rice farmers, low production cost and time, and effectiveness in treatment of foot pronation. Corrective wedges were custom fabricated for each participant based on physical therapy assessment, 3D optical scanning and rapid prototyping techniques. The total time to complete the fabrication process was approximately $6 \mathrm{~h}$, which is practical for custom-made products.

\subsection{Lower extremity alignment}

In line with our hypothesis, results revealed muddy terrain to increase Q-angle for all participants for both $\mathrm{BF}$ and $\mathrm{CI}$ conditions (average increase of deviation of $0.5-2.8$ degrees), likely due to the slippery surface posing a greater demand to control leg alignment. This situation may lead to postural instability and fatigue, therefore increasing foot and knee pain [32]. Consequently, a design of corrective insoles for individuals that primarily work in muddy terrain may require additional wedge height to compensate for increase in lower extremity deviations.

When standing barefoot (the BF condition), participants showed abnormal alignments of the lower extremities on both flat rigid ground and in muddy terrain. In agreement with expectation, when participants were equipped with corrective medial wedge insoles (CI condition), all lower extremity alignments showed less deviation (average improvement of 5.5-16.1 degrees) and could be identified as normal. However, the reduction of extremity alignment might be excessive due to a lack of body movement and the rigid material (PLA) used for fabrication of the insoles. Moreover, the plastic wrap used to secure the insoles might have provided additional support over and above the corrective insoles, possibly altering the results of lower extremity alignment.

\subsection{Lower extremity muscle activity}

Results demonstrate that the muddy terrain resulted in greater activity of both invertor and evertor muscles, which indicates an attempt to increase muscular force to compensate for instability when standing in mud. These findings correspond with the greater deviations observed in Q-angle alignment for participants standing in muddy terrain, as compared with rigid terrain. Additional muscular support might induce fatigue and increase the risk of injury [12].

For both terrain conditions, CIs induced lower TA muscle activity, which was also in agreement with our expectation. An increase in contraction of the TA muscle contributes to deceleration of subtalar joint pronation moment [33]. A decrease in TA muscle activity could be attributed to the $\mathrm{CI}$ intervention reducing pronation of the foot for both rigid and muddy terrain. Although a reduction of TA activity was clearly observed, differences were still relatively small, presumably due to the smooth low-friction surface of the PLA insole. Indeed, a previous study showed insoles with textured surface significantly decreased TA activity during the stance phase [34]. Future development of wedge insoles should investigate the effects of textured surfaces, non-slip pads, or alternative materials on TA activity reduction.

Contrary to expectation, participants exhibited greater PL muscle activity when they stood on a CI as compared with the BF condition. The lower amplitude of the PL may be attributed to slight shortening of the muscle in the pronated position [11], limiting the range of motion and functional movement. The increase in mean activity of the PL muscle might reflect stretching due to a lower degree of foot pronation when supported by a medial wedge insole. A previous study also reported an increase of PL maximum amplitude in asymptomatic participants with a medial wedge assisting lateral ankle stability, as compared to walking with common footwear [35].

\subsection{Study limitations and future research}

A limitation of the current study is that the actual use of the corrective insoles for work will require some method to afix them to the worker's feet (e.g., shoes, sandals, etc.). Such methods may interact with the insoles resulting in forces different than those when just standing on the insoles. Future research should also include development of assistive intervention techniques in the form of footwear for rice farmers, including custom insoles, to be worn while working under various work terrain conditions. 


\section{Conclusions}

This preliminary study demonstrates that corrective insoles, primarily used to support individuals with physical disability, can be extended to help reduce leg malalignments and TA muscle activity, and stretch shortened PL muscles of agricultural workers occupying both rigid and muddy terrains. Results should be used as a basis for future studies on development of corrective insoles for a group of occupational individuals working in a rice paddy, with the objective of identifying broadly effective methods for correcting and preventing common risks of lower extremity injury.

\section{Funding}

This study was supported by a grant from the Thailand Research Fund (TRF) and Office of the Higher Education Commission (OHEC) (No. MRG6080225). The opinions expressed in this paper are those of the authors and do not necessarily reflect the views of TRF or OHEC.

\section{Conflict of interests}

The authors declare that they have no conflict of interests.

\section{References}

[1] Reid CR, McCauley Bush P, Karwowski W, Durrani SK. Occupational postural activity and lower extremity discomfort: a review. Int J Ind Ergon 2010;40(3): $247-56$.

[2] Fathallah FA. Musculoskeletal disorders in labor-intensive agriculture. Appl Ergon 2010;41(6):738-43.

[3] Karukunchit U, Puntumetakul R, Swangnetr M, Boucaut R. Prevalence and risk factor analysis of lower extremity abnormal alignment characteristics among rice farmers. Patient Prefer Adherence 2015;9:785-95.

[4] Horwood AM, Chockalingam N. Defining excessive, over, or hyper-pronation: a quandary. The Foot 2017;31:49-55.

[5] Neubert MS, Karukunchit U, Puntumetakul R. Identification of influential demographic and work-related risk factors associated to lower extremity pain perception among rice farmers. Work Read Mass 2017;58(4):489-98.

[6] Donatelli R, Wooden M. Orthopaedic physical therapy. 4th ed. Elsevier health sciences; 2009.

[7] Juntaracena K, Neubert MS, Puntumetakul R. Effects of muddy terrain on lower extremity muscle activity and discomfort during the rice planting process. Int J Ind Ergon 2018;66:187-93.

[8] Burns J, Keenan A-M, Redmond A. Foot type and overuse injury in triathletes. J Am Podiatr Med Assoc 2005;95(3):235-41.

[9] Yates B, White S. The incidence and risk factors in the development of medial tibia stress syndrome among naval recruits. Am J Sports Med 2004;32(3):772-80.

[10] Franco AH. Pes cavus and pes planus. Analyses and treatment. Phys Ther 1987;67 (5):688-94.

[11] Cobb SC, Tis LL, Johnson BF, Higbie EJ. The effect of forefoot varus on postural stability. J Orthop Sports Phys Ther 2004;34(2):79-85.

[12] Hunt AE, Smith RM. Mechanics and control of the flat versus normal foot during the stance phase of walking. Clin Biomech 2004;19(4):391-7.
[13] Milgrom C, Radeva-Petrova DR, Finestone A, Nyska M, Mendelson S, Benjuya N, et al. The effect of muscle fatigue on in vivo tibial strains. J Biomech 2007;40(4): 845-50.

[14] Mamansari DU, Salokhe VM. The need for ergonomics considerations for the design and development of agricultural machinery in Thailand. J Hum Ergol 1995; 24(1):61-72.

[15] Mohanty SK, Behera BK, Satapathy GC. Ergonomics of farm women in manual paddy threshing. Agric Eng Int CIGR J 2008:X.

[16] Kristanto A, Neubert MS, Puntumetakul R, Sessomboon W. Adaptable ergonomic interventions for patients with cerebral palsy to rice farmers activities: reviews and recommendations. Asia-Pac J Sci Technol 2019;24(4). APST-24-04-10(9 pages).

[17] Landorf KB, Keenan AM. Efficacy of foot orthoses. What does the literature tell us? J Am Podiatr Med Assoc 2000;90(3):149-58.

[18] Yakimovich T, Lemaire ED, Kofman J. Engineering design review of stance-control knee-ankle-foot orthoses. J Rehabil Res Dev 2009;46(2):257-67.

[19] Meyer-Heim A, van Hedel HJA. Robot-assisted and computer-enhanced therapies for children with cerebral palsy: current state and clinical implementation. Semin Pediatr Neurol 2013;20(2):139-45.

[20] Chen MJL, Chen CPC, Lew HL, Hsieh W-C, Yang W-P, Tang SFT. Measurement of forefoot varus angle by laser technology in people with flexible flatfoot. Am J Phys Med Rehabil 2003;82(11):842-6.

[21] Branthwaite HR, Payton CJ, Chockalingam N. The effect of simple insoles on threedimensional foot motion during normal walking. Clin Biomech 2004;19(9):972-7.

[22] Stell JF, Buckley JG. Controlling excessive pronation: a comparison of casted and non-casted orthoses. The Foot 1998;8(4):210-4.

[23] Rizza R, Liu X, Thometz J, Lyon R, Tassone C. A new method in the design of a dynamic pedorthosis for children with residual clubfoot. J Med Devices 2010;4(2).

[24] Jeevana RR, Vijayaragavan E, Kirubac A. 3Dimensional modeling of an ankle foot orthosis for clubfoot deformity. Int J Biomed Res 2011;2(3):171-80.

[25] Tsai L-C, Yu B, Mercer VS, Gross MT. Comparison of different structural foot types for measures of standing postural control. J Orthop Sports Phys Ther 2006;36(12): 942-53.

[26] Magee DJ. Orthopedic physical assessment. 6th ed. London, United Kingdom: Elsevier Health Sciences; 2014.

[27] Solberg G. Postural disorder and musculoskeletal dysfunction: diagnosis, prevention and treatment. 2nd ed. Churchill Livingstone; 2007.

[28] McLoda TA, Hansen AJ, Birrer DA. EMG analysis of peroneal and tibialis anterior muscle activity prior to foot contact during functional activities. Electromyogr Clin Neurophysiol 2004;44(4):223-7.

[29] Smith C, Bushuk M, Appelle J, Jack R, Chen J. Surface electromyographic study of peroneus longus activation during ankle eversion and heel lift. Open J Ther Rehabil 2015;3(3):720-6.

[30] Sousa CDO, Ferreira JJDA, Medeiros ACLV, Carvalho AHD, Pereira RC, Guedes DT, et al. Electromyograhic activity in squatting at 40,60 and 90 knee flexion. positions. Rev Bras Med Esporte 2007;13(5):310-6.

[31] Danielsson A, Willén C, Sunnerhagen KS. Measurement of energy cost by the physiological cost index in walking after stroke. Arch Phys Med Rehabil 2007;88 (10):1298-303.

[32] Nguyen A-D, Shultz SJ. Identifying relationships among lower extremity alignment characteristics. J Athl Train 2009;44(5):511-8.

[33] Kirby KA. Rotational equilibrium across the subtalar joint axis. J Am Podiatr Med Assoc 1989;79(1):1-14.

[34] Nurse MA, Hulliger M, Wakeling JM, Nigg BM, Stefanyshyn DJ. Changing the texture of footwear can alter gait patterns. J Electromyogr Kinesiol Off J Int Soc Electrophysiol Kinesiol 2005;15(5):496-506.

[35] Murley GS, Bird AR. The effect of three levels of foot orthotic wedging on the surface electromyographic activity of selected lower limb muscles during gait. Clin Biomech 2006;21(10):1074-80. 